CERN-PPE/94-58

7 April 1994

\title{
One-Prong $\tau$ Decays into Charged Kaons
}

\author{
The ALEPH Collaboration*)
}

\begin{abstract}
$\underline{\text { Abstract }}$
From a sample of about $75000 \tau$ decays measured in the ALEPH detector, 1-prong charged kaon decays are identified by the $\mathrm{dE} / \mathrm{dx}$ measurement in the central detector. The resulting branching ratios for the inclusive and exclusive modes are : $B\left(\tau \rightarrow \nu_{\tau} K^{-} \geq 0 \pi^{0} \geq 0 K^{0}\right)=(1.60 \pm 0.07 \pm$ $0.12) \%, B\left(\tau \rightarrow \nu_{\tau} K^{-}\right)=(0.64 \pm 0.05 \pm 0.05) \%, B\left(\tau \rightarrow \nu_{\tau} K^{-} \pi^{0}\right)=(0.53 \pm 0.05 \pm 0.07) \%$ and $B\left(\tau \rightarrow \nu_{\tau} K^{-} \pi^{0} \pi^{0}\right)=(0.04 \pm 0.03 \pm 0.02) \%$. Exclusive modes are corrected for measured $K_{L}^{0}$ production. The rate for $\tau \rightarrow \nu_{\tau} K^{-}$agrees well with the prediction based on $\tau-\mu$ universality.
\end{abstract}

(Submitted to Physics Letters B)

*) see next pages for the list of authors 


\section{The ALEPH Collaboration}

D. Buskulic, D. Casper, I. De Bonis, D. Decamp, P. Ghez, C. Goy, J.-P. Lees, M.-N. Minard, P. Odier, B. Pietrzyk

Laboratoire de Physique des Particules (LAPP), IN ${ }^{2} P^{3}-C N R S, 74019$ Annecy-le-Vieux Cedex, France

F. Ariztizabal, M. Chmeissani, J.M. Crespo, I. Efthymiopoulos, E. Fernandez, M. Fernandez-Bosman, V. Gaitan, Ll. Garrido, ${ }^{28}$ M. Martinez, T. Mattison, ${ }^{29}$ S. Orteu, A. Pacheco, C. Padilla, A. Pascual, F. Teubert

Institut de Fisica d'Altes Energies, Universitat Autonoma de Barcelona, 08193 Bellaterra (Barcelona), Spain $^{7}$

D. Creanza, M. de Palma, A. Farilla, G. Iaselli, G. Maggi, N. Marinelli, S. Natali, S. Nuzzo, A. Ranieri, G. Raso, F. Romano, F. Ruggieri, G. Selvaggi, L. Silvestris, P. Tempesta, G. Zito

Dipartimento di Fisica, INFN Sezione di Bari, 70126 Bari, Italy

Y. Chai, D. Huang, X. Huang, J. Lin, T. Wang, Y. Xie, D. Xu, R. Xu, J. Zhang, L. Zhang, W. Zhao Institute of High-Energy Physics, Academia Sinica, Beijing, The People's Republic of China ${ }^{8}$

E. Blucher ${ }^{30}$ G. Bonvicini, J. Boudreau, ${ }^{25}$ P. Comas, P. Coyle, H. Drevermann, R.W. Forty, G. Ganis, C. Gay ${ }^{3}$ M. Girone, R. Hagelberg, J. Harvey, R. Jacobsen, B. Jost, J. Knobloch, I. Lehraus, M. Maggi, C. Markou, P. Mato, H. Meinhard, A. Minten, R. Miquel, P. Palazzi, J.R. Pater, J.A. Perlas, P. Perrodo, J.-F. Pusztaszeri, F. Ranjard, L. Rolandi, J. Rothberg, ${ }^{2}$ T. Ruan, M. Saich, D. Schlatter, M. Schmelling, F. Sefkow, ${ }^{6}$ W. Tejessy, I.R. Tomalin, R. Veenhof, H. Wachsmuth, S. Wasserbaech, ${ }^{2}$ W. Wiedenmann, T. Wildish, W. Witzeling, J. Wotschack

European Laboratory for Particle Physics (CERN), 1211 Geneva 23, Switzerland

Z. Ajaltouni, M. Bardadin-Otwinowska, A. Barres, C. Boyer, A. Falvard, P. Gay, C. Guicheney, P. Henrard, J. Jousset, B. Michel, J-C. Montret, D. Pallin, P. Perret, F. Podlyski, J. Proriol, F. Saadi

Laboratoire de Physique Corpusculaire, Université Blaise Pascal, IN ${ }^{2} P^{3}-C N R S$, ClermontFerrand, 63177 Aubière, France

T. Fearnley, J.B. Hansen, J.D. Hansen, J.R. Hansen, P.H. Hansen, S.D. Johnson, R. Møllerud, B.S. Nilsson

Niels Bohr Institute, 2100 Copenhagen, Denmark ${ }^{9}$

A. Kyriakis, E. Simopoulou, I. Siotis, A. Vayaki, K. Zachariadou

Nuclear Research Center Demokritos (NRCD), Athens, Greece

J. Badier, A. Blondel, G. Bonneaud, J.C. Brient, P. Bourdon, G. Fouque, L. Passalacqua, A. Rougé, M. Rumpf, R. Tanaka, M. Verderi, H. Videau

Laboratoire de Physique Nucléaire et des Hautes Energies, Ecole Polytechnique, IN $\mathrm{N}^{2} \mathrm{P}^{3}$ CNRS, 91128 Palaiseau Cedex, France 
D.J. Candlin, M.I. Parsons, E. Veitch

Department of Physics, University of Edinburgh, Edinburgh EH9 3JZ, United Kingdom ${ }^{10}$

E. Focardi, L. Moneta, G. Parrini

Dipartimento di Fisica, Università di Firenze, INFN Sezione di Firenze, 50125 Firenze, Italy

M. Corden, M. Delfino, ${ }^{12}$ C. Georgiopoulos, D.E. Jaffe, D. Levintha $1^{15}$

Supercomputer Computations Research Institute, Florida State University, Tallahassee, FL 32306-4052, USA ${ }^{13,14}$

A. Antonelli, G. Bencivenni, G. Bologna, ${ }^{4}$ F. Bossi, P. Campana, G. Capon, F. Cerutti, V. Chiarella,

G. Felici, P. Laurelli, G. Mannocchi, ${ }^{5}$ F. Murtas, G.P. Murtas, M. Pepe-Altarelli, S. Salomone

Laboratori Nazionali dell'INFN (LNF-INFN), 00044 Frascati, Italy

P. Colrain, I. ten Have, I.G. Knowles, J.G. Lynch, W. Maitland, W.T. Morton, C. Raine, P. Reeves, J.M. Scarr, K. Smith, M.G. Smith, A.S. Thompson, S. Thorn, R.M. Turnbull

Department of Physics and Astronomy, University of Glasgow, Glasgow G12 8QQ,United Kingdom ${ }^{10}$

U. Becker, O. Braun, C. Geweniger, P. Hanke, V. Hepp, E.E. Kluge, A. Putzer, ${ }^{1}$ B. Rensch, M. Schmidt, H. Stenzel, K. Tittel, M. Wunsch

Institut für Hochenergiephysik, Universität Heidelberg, 69120 Heidelberg, Fed. Rep. of Germany ${ }^{16}$

R. Beuselinck, D.M. Binnie, W. Cameron, M. Cattaneo, D.J. Colling, P.J. Dornan, J.F. Hassard, N. Konstantinidis, A. Moutoussi, J. Nash, D.G. Payne, G. San Martin, J.K. Sedgbeer, A.G. Wright

Department of Physics, Imperial College, London SW7 2BZ, United Kingdom ${ }^{10}$

P. Girtler, D. Kuhn, G. Rudolph, R. Vogl

Institut für Experimentalphysik, Universität Innsbruck, 6020 Innsbruck, Austria $^{18}$

C.K. Bowdery, T.J. Brodbeck, A.J. Finch, F. Foster, G. Hughes, D. Jackson, N.R. Keemer, M. Nuttall, A. Patel, T. Sloan, S.W. Snow, E.P. Whelan

Department of Physics, University of Lancaster, Lancaster LA1 4YB, United Kingdom ${ }^{10}$

A. Galla, A.M. Greene, K. Kleinknecht, J. Raab, B. Renk, H.-G. Sander, H. Schmidt, S.M. Walther, R. Wanke, B. Wolf

Institut für Physik, Universität Mainz, 55099 Mainz, Fed. Rep. of Germany ${ }^{16}$

A.M. Bencheikh, C. Benchouk, A. Bonissent, D. Calvet, J. Carr, C. Diaconu, F. Etienne, D. Nicod, P. Payre, L. Roos, D. Rousseau, P. Schwemling, M. Talby

Centre de Physique des Particules, Faculté des Sciences de Luminy, IN ${ }^{2} P^{3}-C N R S, 13288$

Marseille, France 
S. Adlung, R. Assmann, C. Bauer, W. Blum, D. Brown, P. Cattaneo, ${ }^{23}$ B. Dehning, H. Dietl, F. Dydak, ${ }^{21}$ M. Frank, A.W. Halley, K. Jakobs, H. Kroha, J. Lauber, G. Lütjens, G. Lutz, W. Männer, H.-G. Moser, R. Richter, J. Schröder, A.S. Schwarz, R. Settles, H. Seywerd, U. Stierlin, U. Stiegler, R. St. Denis, G. Wolf

Max-Planck-Institut für Physik, Werner-Heisenberg-Institut, 80805 München, Fed. Rep. of Germany ${ }^{16}$

R. Alemany, J. Boucrot, O. Callot, A. Cordier, M. Davier, L. Duflot, J.-F. Grivaz, Ph. Heusse, P. Janot, D.W. Kim ${ }^{19}$ F. Le Diberder, J. Lefrançois, A.-M. Lutz, G. Musolino, H.-J. Park, M.-H. Schune, J.J. Veillet, I. Videan

Laboratoire de l'Accélérateur Linéaire, Université de Paris-Sud, IN $\mathrm{N}^{2} \mathrm{P}^{3}$ CNRS, 91405 Orsay Cedex, France

D. Abbaneo, G. Bagliesi, G. Batignani, U. Bottigli, C. Bozzi, G. Calderini, M. Carpinelli, M.A. Ciocci, V. Ciulli, R. Dell'Orso, I. Ferrante, F. Fidecaro, L. Foà, ${ }^{1}$ F. Forti, A. Giassi, M.A. Giorgi, A. Gregorio, F. Ligabue, A. Lusiani, P.S. Marrocchesi, E.B. Martin, A. Messineo, F. Palla, G. Rizzo, G. Sanguinetti, P. Spagnolo, J. Steinberger, R. Tenchini, ${ }^{1}$ G. Tonelli, ${ }^{27}$ G. Triggiani, A. Valassi, C. Vannini, A. Venturi, P.G. Verdini, J. Walsh

Dipartimento di Fisica dell'Università, INFN Sezione di Pisa, e Scuola Normale Superiore, 56010 Pisa, Italy

A.P. Betteridge, Y. Gao, M.G. Green, D.L. Johnson, P.V. March, T. Medcalf, L1.M. Mir, I.S. Quazi, J.A. Strong

Department of Physics, Royal Holloway \& Bedford New College, University of London, Surrey TW20 OEX, United Kingdom ${ }^{10}$

V. Bertin, D.R. Botterill, R.W. Clifft, T.R. Edgecock, S. Haywood, M. Edwards, P.R. Norton, J.C. Thompson

Particle Physics Dept., Rutherford Appleton Laboratory, Chilton, Didcot, Oxon OX11 OQX, United Kingdom ${ }^{10}$

B. Bloch-Devaux, P. Colas, H. Duarte, S. Emery, W. Kozanecki, E. Lançon, M.C. Lemaire, E. Locci, B. Marx, P. Perez, J. Rander, J.-F. Renardy, A. Rosowsky, A. Roussarie, J.-P. Schuller, J. Schwindling, D. Si Mohand, B. Vallage

Service de Physique des Particules, DAPNIA, CE-Saclay, 91191 Gif-sur-Yvette Cedex, France $^{17}$

R.P. Johnson, A.M. Litke, G. Taylor, J. Wear

Institute for Particle Physics, University of California at Santa Cruz, Santa Cruz, CA 95064 , $U_{S A}{ }^{22}$

A. Beddall, C.N. Booth, S. Cartwright, F. Combley, I. Dawson, A. Koksal, C. Rankin, L.F. Thompson Department of Physics, University of Sheffield, Sheffield S3 7RH, United Kingdom ${ }^{10}$

A. Böhrer, S. Brandt, G. Cowan, ${ }^{1}$ E. Feigl, C. Grupen, G. Lutters, J. Minguet-Rodriguez, F. Rivera, ${ }^{26}$ P. Saraiva, U. Schäfer, L. Smolik

Fachbereich Physik, Universität Siegen, 57068 Siegen, Fed. Rep. of Germany ${ }^{16}$ 
L. Bosisio, R. Della Marina, G. Giannini, B. Gobbo, L. Pitis, F. Ragusa ${ }^{20}$

Dipartimento di Fisica, Università di Trieste e INFN Sezione di Trieste, 34127 Trieste, Italy

L. Bellantoni, W. Chen, J.S. Conway, ${ }^{24}$ Z. Feng, D.P.S. Ferguson, Y.S. Gao, J. Grahl, J.L. Harton, O.J. Hayes, H. Hu, J.M. Nachtman, Y.B. Pan, Y. Saadi, M. Schmitt, I. Scott, V. Sharma, J.D. Turk, A.M. Walsh, F.V. Weber, Sau Lan Wu, X. Wu, J.M. Yamartino, M. Zheng, G. Zobernig

Department of Physics, University of Wisconsin, Madison, WI 53706, USA ${ }^{11}$

\footnotetext{
${ }^{1}$ Now at CERN, PPE Division, 1211 Geneva 23, Switzerland.

${ }^{2}$ Permanent address: University of Washington, Seattle, WA 98195, USA.

${ }^{3}$ Now at Harvard University, Cambridge, MA 02138, U.S.A.

${ }^{4}$ Also Istituto di Fisica Generale, Università di Torino, Torino, Italy.

${ }^{5}$ Also Istituto di Cosmo-Geofisica del C.N.R., Torino, Italy.

${ }^{6}$ Now at DESY, Hamburg, Germany.

${ }^{7}$ Supported by CICYT, Spain.

${ }^{8}$ Supported by the National Science Foundation of China.

${ }^{9}$ Supported by the Danish Natural Science Research Council.

${ }^{10}$ Supported by the UK Science and Engineering Research Council.

${ }^{11}$ Supported by the US Department of Energy, contract DE-AC02-76ER00881.

${ }^{12}$ On leave from Universitat Autonoma de Barcelona, Barcelona, Spain.

${ }^{13}$ Supported by the US Department of Energy, contract DE-FG05-92ER40742.

${ }^{14}$ Supported by the US Department of Energy, contract DE-FC05-85ER250000.

${ }^{15}$ Present address: Lion Valley Vineyards, Cornelius, Oregon, U.S.A.

${ }^{16}$ Supported by the Bundesministerium für Forschung und Technologie, Fed. Rep. of Germany.

${ }^{17}$ Supported by the Direction des Sciences de la Matière, C.E.A.

${ }^{18}$ Supported by Fonds zur Förderung der wissenschaftlichen Forschung, Austria.

${ }^{19}$ Permanent address: Kangnung National University, Kangnung, Korea.

${ }^{20}$ Now at Dipartimento di Fisica, Università di Milano, Milano, Italy.

${ }^{21}$ Also at CERN, PPE Division, 1211 Geneva 23, Switzerland.

${ }^{22}$ Supported by the US Department of Energy, grant DE-FG03-92ER40689.

${ }^{23}$ Now at Università di Pavia, Pavia, Italy.

${ }^{24}$ Now at Rutgers University, Piscataway, N.J 08854, USA.

${ }^{25}$ Now at University of Pittsburgh, Pittsburgh, PA 15260, U.S.A.

${ }^{26}$ Partially supported by Colciencias, Colombia.

${ }^{27}$ Also at Istituto di Matematica e Fisica, Università di Sassari, Sassari, Italy.

${ }^{28}$ Permanent address: Dept. d'Estructura i Constituens de la Materia, Universitat de Barcelona, 08208 Barcelona, Spain.

${ }^{29}$ Now at SLAC, Stanford, CA 94309 , U.S.A.

${ }^{30}$ Now at University of Chicago, Chicago, IL 60637, U.S.A.
} 


\section{Introduction}

The experimental situation in kaon production in $\tau$ decays is still unsatisfactory[1]. Except for $\mathrm{K}^{*}$ production, which has been studied through the $\left(\tau \rightarrow \nu_{\tau} \mathrm{K}^{*}, \mathrm{~K}^{*} \rightarrow \mathrm{K}_{S}^{0} \pi^{-}, \mathrm{K}_{S}^{0} \rightarrow \pi^{+} \pi^{-}\right)$decay chain, the rates for the other Cabibbo-suppressed channels are not very well known. In particular, the measurements of the simplest process

$$
\tau \rightarrow \nu_{\tau} K^{-}
$$

which are few and suffer from low statistics[2,3] do not permit a precise test of the $\tau-\mu$ universality by comparison to the leptonic rate for $K^{-} \rightarrow \bar{\nu}_{\mu} \mu^{-}$.

Beyond the K and $\mathrm{K}^{*}$ channels, additional decay modes with more pions are expected, but they have not yet been investigated experimentally. It is also possible to consider Cabibbo-allowed decays with a pair of kaons: such modes are expected to be at a small level although no reliable theoretical estimate is available. Since some of these final states can involve a charged kaon and a neutral kaon which was often not detected in previous experiments, it is worthwhile to undertake a systematic study of kaon production in $\tau$ decays.

Such an investigation of 1-prong $\tau$ decays with the ALEPH detector is described in this letter and the accompanying one [4]. Hadronic $\tau$ decays are classified according to their $\pi^{0}$ multiplicity (up to two $\pi^{0}$ 's) and the charged $\mathrm{K}$ fraction is determined using a $\mathrm{dE} / \mathrm{dx}$ measurement in the central detector (this letter). A complementary search for final states containing $\mathrm{K}^{0}$ 's is performed using hadronic calorimetry, which is sensitive to the $\mathrm{K}_{L}^{0}$ component (the following letter). Combining the two analyses allows separation of the various exclusive decay channels and the measurement of the branching ratios for the dominant modes.

In this letter, ALEPH measurements of the 1-prong charged kaon $\tau$-decay rates are presented, i.e.

$$
\tau \rightarrow \nu_{\tau} K^{-} \geq 0 \text { neutrals }
$$

where the neutrals include $\pi^{0}$ s and $\mathrm{K}^{0}$ 's. The branching fractions for the channels

$$
\begin{aligned}
\tau & \rightarrow \nu_{\tau} K^{-} \\
\tau & \rightarrow \nu_{\tau} K^{-} \pi^{0} \\
\tau & \rightarrow \nu_{\tau} K^{-} \pi^{0} \pi^{0}
\end{aligned}
$$

are extracted, after subtracting small contributions from other decay modes $\left(\tau \rightarrow \nu_{\tau} K^{-} K^{0}\right.$ and $\tau \rightarrow$ $\nu_{\tau} K^{-} \pi^{0} K^{0}$ ) measured with the calorimetric analysis[4]. 


\section{The ALEPH detector and the ionization measurement}

A detailed description of the ALEPH detector can be found elsewhere[5]. Charged particle momenta are measured by a magnetic spectrometer consisting of a precision vertex detector (VDET), of a cylindrical drift chamber, the inner tracking chamber (ITC), and of a large time projection chamber (TPC). The 1.5 $\mathrm{T}$ axial magnetic field is provided by a superconducting solenoidal coil. The transverse momentum resolution is $\sigma_{p} / \mathrm{p}=6 \times 10^{-4} \mathrm{p}$, with $\mathrm{p}$ in $\mathrm{GeV} / \mathrm{c}$. Photons are measured with an energy resolution $\sigma_{E} / \mathrm{E}=0.18 / \sqrt{E}$, with $\mathrm{E}$ in $\mathrm{GeV}$, in the electromagnetic calorimeter (ECAL), a 22 radiation length thick sandwich of lead plates and proportional wire chambers. The fine solid-angle readout segmentation $\left(1^{0} \times 1^{0}\right)$ is essential for high-energy $\pi^{0}$ reconstruction and the 3 -fold longitudinal segmentation allows a check of the electromagnetic nature of the detected showers. The $1.2 \mathrm{~m}$ thick iron return yoke of the magnet is interleaved with 23 layers of streamer tubes and acts as a hadronic calorimeter (HCAL).

In the data sample used for this analysis, all components of the detector were required to be operational. Furthermore, one of the following trigger conditions was demanded: a minimum ECAL energy of $6 \mathrm{GeV}$, or a track segment in the ITC pointing to an energy deposit in ECAL of a least $1.2 \mathrm{GeV}$, or a track segment in the ITC matching the signal of a penetrating particle in HCAL.

Of prime interest for this analysis is the measurement of the ionization deposited by charged particles traversing the TPC gas at atmospheric pressure and sampled up to 330 times on the sense wires of the TPC end-plates. To avoid the large fluctuations caused by $\delta$-rays, a truncated mean is used, leaving aside the $40 \%$ largest values. The resulting resolution is $5.0 \%$ for tracks at a polar angle of $90^{\circ}$, decreasing to $4.0 \%$ near $40^{\circ}$ where the track length is maximum and increasing again to $5.3 \%$ down to $20^{\circ}$ because of the reduced number of samples.

\section{Event selection}

The present analysis is based on a data sample corresponding to about one million hadronic $\mathrm{Z}$ decays collected by ALEPH in 1991 and 1992. Candidates for the $\tau^{+} \tau^{-}$final states are selected using an off-line filter, designed to reduce backgrounds with specific cuts while preserving $\tau^{+} \tau^{-}$candidates in background-free topologies[6]. This goal is achieved using particle identification to reduce Bhabha, $\mu-$ pair and $\gamma \gamma$-induced lepton pair backgrounds and applying multiplicity cuts to suppress hadronic Z decays.

Within the geometrical acceptance of $84.2 \%$ defined for a reliable analysis, the $\tau^{+} \tau^{-}$filter has an efficiency of $92.8 \%$, with a background contamination of only $1.6 \%$, the largest contribution coming from Bhabha events $(0.7 \%)$. The one-prong hadronic $\tau$ decay sample studied is even cleaner with a non- $\tau$ background of $0.1 \%$.

Decays with only one charged particle originating from the interaction region are selected by requiring a minimum momentum of $2 \mathrm{GeV} / \mathrm{c}$ and a positive hadron identification. The latter requirement is based on a likelihood method using ECAL and HCAL information, as described in Ref. 7. In the selected momentum range, hadrons are identified with an efficiency of $98.5 \%$ ( $0.9 \%$ of them being mis-identified as muons). The electron and muon contaminations in the hadron sample are $0.18 \%$ and $0.22 \%$, respectively. 
Next, photons are identified in ECAL with an energy threshold of $250 \mathrm{MeV}$ and $\pi^{0}$ 's are reconstructed with a cut on the $\gamma \gamma$ invariant mass $\left(0.07-0.21 \mathrm{GeV} / \mathrm{c}^{2}\right)$. Since high-energy $\pi^{0}$ decays cannot be easily resolved and low-energy photons are lost, single photons with an energy in excess of $4 \mathrm{GeV}$ are also retained as $\pi^{0}$ candidates[8]. Four data samples are defined with respect to the $\pi^{0}$ multiplicity : the global sample, with no requirement on the number of $\pi^{0}$ 's, is referred to as 'h inclusive', whereas three sub-samples, ' $h$ ', ' $h \pi^{0}$, ' $h 2 \pi^{0}$, are labelled by the observed number of $\pi^{0}$ 's. Events with additional photons are excluded from these exclusive samples. The efficiencies of this classification for kaon final states are calculated by Monte Carlo[9] to be $84 \%, 56 \%$ and $39 \%$ for the 'h', 'h $\pi^{0}$ ' and 'h $2 \pi^{0}$ ' samples, respectively, while the corresponding purities (fraction of events in a given class originating from the correct $\pi^{0}$ multiplicity) are $98 \%, 96 \%$ and $82 \%$.

The $\tau^{+} \tau^{-}$filter selects a sample of 37778 events, yielding 28481 one-prong hadronic $\tau$ candidates after all cuts, of which 28390 have $\mathrm{dE} / \mathrm{dx}$ information.

\section{$4 \mathrm{~K} / \pi$ separation by $\mathrm{dE} / \mathrm{dx}$ and calibration}

The $\mathrm{dE} / \mathrm{dx}$ measured in the TPC (hereafter called $\mathrm{R}$ ) is to a very good approximation Gaussiandistributed around the expected value $\mathrm{R}_{i}$ for a given particle type $i$. In the standard ALEPH analyses using $\mathrm{dE} / \mathrm{dx}$, the $\mathrm{R}_{i}$ values are computed using a parametrization of the Bethe-Bloch formula fitted to ALEPH data[10]. The calculations of $\mathrm{R}_{i}$ and its variance $\sigma_{i}^{2}$ take into account efficiencies and geometrical effects, through the number of samplings and the track sample length, which are both angle-dependent and include the effect of the small dead zones between TPC sectors in the end-plates.

Taking these estimates of $\mathrm{R}_{i}$ and $\sigma_{i}$ as a starting point, a finer calibration is performed, taking into account the following facts :

(i) the standard calibration, obtained mostly from pions in hadronic $\mathrm{Z}$ decays corresponding to tracks in general not isolated in the TPC, is not precise enough for the purpose of this analysis where the kaon fraction is expected to be low (a few \%).

(ii) one-prong $\tau$ decays offer a unique possibility to achieve a better calibration : in contrast to the $\mathrm{Z}$ hadronic sample, tracks are completely isolated and unperturbed by the rest of the event. Furthermore, $\mathrm{Z}$ and $\tau$ decays into muons (in the range from 2 to $45 \mathrm{GeV} / \mathrm{c}$ ) can be used with the advantage of the same angular distribution in the detector as for the hadrons under study. For the sample of $\tau$ decays into muons, it is important to take into account the small contributions from hadrons mis-identified as muons.

Thus the shape of the $\mathrm{R}$ distribution is taken from muons. The distribution $\rho\left(x_{\mu}\right)$ of the quantity

$$
x_{\mu}=\frac{R-R_{\mu}}{\sigma_{\mu}}
$$

deviates slightly from a pure Gaussian with zero mean and unit variance and a more precise parametrization is obtained from the muon samples. No significant dependence of $\rho\left(x_{\mu}\right)$ on momentum is noticed in the $2-45 \mathrm{GeV} / \mathrm{c}$ range. The muon $\mathrm{R}$ probability density is therefore given by

$$
W_{\mu}(R)=\frac{\rho\left(x_{\mu}\right)}{\sigma_{\mu}}
$$


with $x_{\mu}$ defined by (4). The densities $W_{i}(R)$ for the other particle types are obtained from the same $\rho$ distribution and from the calibrated $\mathrm{R}_{i}$ and $\sigma_{i}$.

The $\mathrm{K}$ fraction $\mathrm{f}_{K}$ is obtained from the distribution of $x_{\pi}$ (defined analogously to (4)) for the hadron sample which is fitted to a sum of $\pi$ and $\mathrm{K}$ contributions, each proportional to $\mathrm{W}_{\pi, K}(R)$, respectively. Another approach is to define a kaon estimator for each hadron with ionization $\mathrm{R}$ :

$$
P_{K}=\frac{W_{K}(R)}{W_{\pi}(R)+W_{K}(R)}
$$

A peak near one in the distribution of this variable signals the presence of kaons.

The $\mathrm{K} / \pi$ separation is momentum-dependent. The variation of the separation parameter

$$
\eta=\frac{R_{\pi}-R_{K}}{\sigma_{\pi}}
$$

is given in Figure 1.

\section{$5 \quad$ Fits and systematic uncertainties}

To derive the $\mathrm{K}$ fraction in each of the data samples, the respective $x_{\pi}$ distributions are not only fitted to a linear combination of pion and kaon expected contributions, but also to small components for electrons and muons. This latter contribution does not significantly affect the derived K fraction, but improves the overall description of the ionization loss distribution. The rates for these contaminations are kept fixed, and are given by the Monte Carlo simulation of the particle identification which has been checked with real data. The fits are performed with both unbinned likelihood or binned $\chi^{2}$ methods : both procedures yield consistent results and statistical errors. The results quoted below are from the $\chi^{2}$ fits.

The fit results for the inclusive hadron sample are given in Figure 2. A significant contribution from kaons is observed corresponding to a clear peak at one in the $\mathrm{P}_{K}$ distribution. Similar excesses are observed in all hadron samples.

Beyond statistical limitations the main source of uncertainty is the description of the pion $\mathrm{dE} / \mathrm{dx}$ distribution as a function of momentum. An estimate of the systematic uncertainty involved in this procedure is obtained from fitting a hypothetical $\mathrm{K}$ fraction in the muon samples. The distribution of identified muons in $\tau$ decays is dominated by real muons with a pion contamination taken from the Monte Carlo simulation of the particle identification. A kaon component is left free in the fit (Fig. 3) which yields a fraction $((0.14 \pm 0.06) \%)$ compatible with the expected kaon mis-identification predicted by the Monte Carlo $((0.11 \pm 0.02) \%)$. Fixing this real kaon contribution at the expected value, fits are performed in different momentum bins of the same muon sample searching for an additional (fake) kaon signal located at $R=R_{K}+R_{\mu}-R_{\pi}$. Such a procedure is sensitive to a local distortion in the reference distribution $\rho(x)$ measured with muons, which would reflect into a fake K signal in the hadron $x_{\pi}$ distribution.

The results of this procedure are given in Table 1 : the values are consistent with zero and they are used to bound possible systematic effects through convolution with the momentum distribution of each 
hadron sample. The resulting absolute systematic uncertainty on the kaon fraction $f_{K}$ is estimated to be $0.22 \%$, almost independent of the data sample.

The calibrations of $\mathrm{R}$ and $\sigma$ can be checked using the hadron sample. A likelihood fit with 3 parameters ( $\mathrm{K}$ fraction, deviation $\Delta \mathrm{R}$ from expected mean $\mathrm{R}_{\pi}$ value and ratio $\lambda$ of the standard deviation to the expected value $\sigma_{\pi}$ ), yields

$$
\begin{aligned}
\Delta R & =-0.00072 \pm 0.00084 \\
\lambda & =1.0014 \pm 0.0097
\end{aligned}
$$

The values are consistent with the calibration based on muons and show that the knowledge of the absolute ionization loss scale is at the $0.1 \%$ level, while the standard deviation is known to about $1 \%$.

Additional checks are possible :

(i) Because the large kaon mass cannot be neglected in $\tau$ decays, the $\mathrm{K}$ fraction is expected to vanish below the kinematic threshold of $3.6 \mathrm{GeV} / \mathrm{c}$ (except for a very small contribution from radiative events). In the range $2-3.5 \mathrm{GeV} / \mathrm{c}$, the inclusive sample with $f_{K}=(0.08 \pm 0.34) \%$ is consistent with the Monte Carlo expectation, $f_{K}^{M C}=(0.05 \pm 0.03) \%$. This test is less precise than the quoted systematic uncertainty, but it is consistent and completely independent.

(ii) The $\mathrm{K} \pi^{0}$ channel should be dominated by the $\mathrm{K}^{*}(892)$ resonance, characterized by a smaller $\mathrm{Q}$ value compared to the $\rho \rightarrow \pi \pi^{0}$. A cut on the $\mathrm{K} \pi$ mass below $1 \mathrm{GeV} / \mathrm{c}^{2}$ isolates most of the $\mathrm{K}^{*}$ events, hence the $\mathrm{K}$ sample. Defining $r$ as the ratio of the $\mathrm{f}_{K}$ values above and below the cut, a good agreement is observed between data and Monte Carlo with $r$ values of $(8 \pm 3) \%$ and $(8.6 \pm 1.0) \%$, respectively. If the measured $f_{K}$ were due to fake kaons $r$ would be consistent with unity. While this test is a factor of two more precise than the quoted systematics, it relies on $\mathrm{K}^{*}$ dominance. So the more conservative estimate is preferred.

Less important contributions to the systematic uncertainty are estimated, such as those related to the selection efficiency of a given channel and its purity. The relative uncertainties for this source vary from 2 to $4 \%$, depending on the sample. The final values for systematic uncertainties are quoted with the final results in Table 2.

\section{Discussion of the results}

The K fractions, as obtained by the fits, are given in Table 2. The quality of the fits is good and the resulting statistical power is close to that of an ideal analysis identifying kaons perfectly on an event-byevent basis, the sensitivity being degraded by only a factor of 0.75 .

Given the selection procedure, it is possible that additional $\mathrm{K}^{0}$ 's could be present in the same $\tau$ hemisphere. Almost all $\mathrm{K}_{S}^{0}$ decays to $\pi^{+} \pi^{-}$would have at least one extra track pointing to the interaction vertex and hence the efficiency for keeping these decays is very small. Most $\mathrm{K}^{0}$ contamination would 
therefore come from $\mathrm{K}_{S}^{0}$ 's decaying into $\pi^{0} \pi^{0}, \mathrm{~K}_{S}^{0}$ 's not decaying in the TPC and $\mathrm{K}_{L}^{0}$ 's. These contributions can be estimated with the KORALZ Monte Carlo[9] up to a scale factor, since the decay modes in question are not yet experimentally measured. In addition, some possible decay channels like

$$
\tau \rightarrow \nu_{\tau} K^{-} K^{0}
$$

are not yet simulated in KORALZ and are treated separately in a model assuming a spin-one state for the $\mathrm{K}^{-} \mathrm{K}^{0}$ system ${ }^{1}$.

By performing a dedicated complementary search for $\mathrm{K}_{L}^{0}$ candidates in $\mathrm{HCAL}$, these contributions can be evaluated directly from the data. Searching for $\mathrm{K}_{L}^{0}$ 's rather than $\mathrm{K}_{S}^{0}$ 's has the advantage of being completely independent of the present analysis using tracks (TPC) and photons (ECAL). The $\mathrm{K}_{L}^{0}$ analysis is described separately[4] and studies the decay modes

$$
\tau \rightarrow \nu_{\tau} h^{-} K^{0}
$$

and

$$
\tau \rightarrow \nu_{\tau} h^{-} \pi^{0} K^{0}
$$

Contamination of the present event samples by the $\mathrm{K}^{-} \mathrm{K}^{0}$ and $\mathrm{K}^{-} \pi^{0} \mathrm{~K}^{0}$ channels are computed using the Monte Carlo simulation normalized to the measured total rate in the $\mathrm{K}_{L}^{0}$ analysis[4]. The corresponding values are used to correct the measured branching ratios into the final states of interest for the present analysis.

The final results are given in Table 2. For each channel, the efficiency and the background are computed with the Monte Carlo and the corrections for $\mathrm{K}^{0}$ production $\left(\Delta N_{K}\right)$ are indicated. The latter have a different meaning for the different channels : in the inclusive mode (2) $\Delta N_{K}$ is the loss of events with $\mathrm{K}_{S}^{0}$ 's decaying to $\pi^{+} \pi^{-}$, whereas for the exclusive modes (3) it refers to the background from channels with additional $\mathrm{K}^{0}$ 's.

The total one-prong inclusive $\mathrm{K}$ rate

$$
B\left(\tau \rightarrow \nu_{\tau} K^{-} \geq 0 \pi^{0} \geq 0 K^{0}\right)=(1.60 \pm 0.07 \pm 0.12) \%
$$

agrees well with the world average[11], $(1.68 \pm 0.24) \%$. However the treatment of $\mathrm{K}^{0}$ 's is explicit in this analysis.

The improvement in the single K channel is significant with

$$
B\left(\tau \rightarrow \nu_{\tau} K^{-}\right)=(0.64 \pm 0.05 \pm 0.05) \%
$$

to be compared to the world average[11], $(0.67 \pm 0.23) \%$, which was not corrected for $\mathrm{K}^{0}$ production. A test of the Standard Model can be performed in the strange sector of the $\tau$ : using the electron branching ratio from Ref. 7 , the ratio

$$
\frac{B\left(\tau \rightarrow \nu_{\tau} K^{-}\right)}{B\left(\tau \rightarrow \nu_{\tau} e \bar{\nu}_{e}\right)}=0.035 \pm 0.004
$$

can be compared to the value of $(0.0393 \pm 0.0002)$ expected from $\tau-\mu$ universality, the measured $\mathrm{K} \rightarrow \mu \nu$ decay rate[11] and radiative corrections[12]. Fitting the $\mathrm{K}$ fraction in each momentum bin, the $\mathrm{K}$ momentum spectrum agrees with the expected distribution including the effect of $\tau$ polarisation.

\footnotetext{
${ }^{1}$ A spin- 0 state would correspond to a second-class current in $\tau$ decay.
} 
The observed branching ratios for the other modes are

$$
\begin{aligned}
B\left(\tau \rightarrow \nu_{\tau} K \pi^{0}\right) & =(0.53 \pm 0.05 \pm 0.07) \% \\
B\left(\tau \rightarrow \nu_{\tau} K \pi^{0} \pi^{0}\right) & =(0.04 \pm 0.03 \pm 0.02) \%
\end{aligned}
$$

The $\mathrm{K}^{*}(892)$ is expected to dominate the $\tau \rightarrow \nu_{\tau} K \pi$ channel. Previous measurements have studied the mode $\mathrm{K}_{S}^{0} \pi^{-}$, with $\mathrm{K}_{S}^{0} \rightarrow \pi^{+} \pi^{-}$. The present result for the $\mathrm{K} \pi^{0}$ mode is consistent with the world average using $\mathrm{K}_{S}^{0} \pi^{-}$, with a comparable uncertainty. The $\mathrm{K} \pi^{0}$ mass distribution, obtained from separate $\mathrm{dE} / \mathrm{dx}$ fits in each mass bin, is well-described by the $\mathrm{K}^{*}$ resonance (Fig. 4 ) and other small non-K $\pi^{0}$ contributions obtained by KORALZ. The fitted mass is $903 \pm 8 \mathrm{MeV}$, in good agreement with the standard value[11], while the fitted resolution of $46 \pm 5 \mathrm{MeV}$ matches the Monte Carlo value of $48 \pm$ $2 \mathrm{MeV}$. The branching ratio for $\tau \rightarrow \nu_{\tau} \mathrm{K}^{*}$ is given in the following letter[4] taking into account the measurements of the $\mathrm{K} \pi^{0}$ and $\mathrm{K}^{0} \pi$ final states.

Finally, the sum of the exclusive modes considered here and in Ref. 4 saturates the one-prong inclusive $\mathrm{K}$ rate within experimental accuracy, thus ruling out other exclusive channels (with more $\pi^{0}$ 's or more $\mathrm{K}^{0} \mathrm{~s}$ ) at the $0.2 \%$ level :

$$
B_{K \text { incl }}-\left(B_{K}+B_{K K^{0}}+B_{K \pi^{0}}+B_{K \pi^{0} K^{0}}+B_{K \pi^{0} \pi^{0}}\right)=(0.05 \pm 0.13) \%
$$

\section{Summary}

Using $\mathrm{dE} / \mathrm{dx}$ measurements in the ALEPH TPC, $\mathrm{K}-\pi$ separation at more than 2 standard deviations is possible over almost the full momentum range. The inclusive rate of one-prong $\tau$ decays with a charged kaon is measured. Using a classification of final states with $\pi^{0}$ reconstruction and a complementary calorimetric method of $\mathrm{K}_{L}^{0}$ detection[4], several exclusive decay modes are identified and measured for the first time:

$$
\begin{aligned}
B\left(\tau \rightarrow \nu_{\tau} K^{-} \geq 0 \pi^{0} \geq K^{0}\right) & =(1.60 \pm 0.07 \pm 0.12) \% \\
B\left(\tau \rightarrow \nu_{\tau} K^{-}\right) & =(0.64 \pm 0.05 \pm 0.05) \% \\
B\left(\tau \rightarrow \nu_{\tau} K^{-} \pi^{0}\right) & =(0.53 \pm 0.05 \pm 0.07) \% \\
B\left(\tau \rightarrow \nu_{\tau} K^{-} \pi^{0} \pi^{0}\right) & =(0.04 \pm 0.03 \pm 0.02) \%
\end{aligned}
$$

These results agree with previous measurements when available $[2,3,13,14]$, but are significantly more precise. 


\section{Acknowledgments}

We wish to thank our colleagues from the accelerator divisions for the successful operation of LEP. We are indebted to the engineers and technicians in all our institutions for their contribution to the excellent performance of ALEPH. Those of us from non-member countries thank CERN for its hospitality.

\section{References}

[1] For a recent review see: M. Davier, Proceedings of the 2nd International Workshop on $\tau$ Lepton Physics (Colombus 1992), K.K. Gan ed., World Scientific (1993)

[2] C.A. Blocker et al., Mark II Coll., Phys. Rev. Lett. 48 (1982) 1586

[3] G.B. Mills et al., DELCO Coll., Phys. Rev. Lett. 52 (1984) 1944

[4] D. Buskulic et al., ALEPH Coll., following letter, preprint CERN-PPE/94-059

[5] D. Décamp et al., ALEPH Coll., Nucl. Inst. and Methods A294 (1990) 121

[6] D. Buskulic et al., ALEPH Coll., CERN-PPE/94-030 (Feb. 94), to be published in Z. Phys.

[7] D. Décamp et al., ALEPH Coll., Z. Phys. C54 (1992) 211

[8] D. Buskulic et al., ALEPH Coll., Z. Phys. C59 (1993) 369

[9] S. Jadach, B.F.L. Ward, and Z. Was, Comp. Phys. Comm. 66(1991)276. KORALZ version 3.8; S. Jadach, J.H. Kuhn, and Z. Was, Comp. Phys. Comm. 64(1991)275.

[10] D. Buskulic et al., ALEPH Coll., Phys. Lett. B303 (1993) 198 ;

W. Atwood et al., Nucl. Instrum. Meth. A306 (1991) 446

[11] Review of Particle Properties, Phys. Rev. D45 (1992)

[12] W.J. Marciano and A. Sirlin, Phys. Rev. Lett. 61 (1988) 1815

[13] Preliminary results for some of these channels from DELPHI and OPAL collaborations have been presented : A. Schwarz, XVI-th International Symposium on Lepton-Photon Interactions, Cornell University (August 93)

[14] M. Battle et al., CLEO Coll., Measurement of Cabibbo-suppressed decays of the $\tau$ lepton, Cornell University preprint CLNS 94/1273 (Feb.94) 


\begin{tabular}{|c|c|c|}
\hline muon source & $\begin{array}{c}\text { momentum range } \\
(\mathrm{GeV} / \mathrm{c})\end{array}$ & $\mathrm{f}_{K}(\%)$ \\
\hline$\tau \rightarrow \nu_{\tau} \mu \bar{\nu}_{\mu}$ & $2-5$ & $0.10 \pm 0.39$ \\
& $5-10$ & $-0.29 \pm 0.21$ \\
& $10-20$ & $0.14 \pm 0.17$ \\
\hline $\mathrm{Z}^{0} \rightarrow \mu^{+} \mu^{-}$ & 45.6 & $0.02 \pm 0.22$ \\
\hline
\end{tabular}

Table 1: Fake $\mathrm{K}$ fractions $\mathrm{f}_{K}$ used to estimate global systematic uncertainties: in the case of muons from $\tau$ decays, the mis-identification of kaons as muons is taken into account in the fit and is not counted as a signal. 


\begin{tabular}{|c|c|c|c|c|}
\hline sample & 'h inclusive' & ' $\mathrm{h}$, & 'h $\pi^{0}$, & 'h $2 \pi^{0}$, \\
\hline decays & 28390 & 6764 & 11503 & 2788 \\
$\mathrm{f}_{K}(\%)$ & $3.17 \pm 0.14$ & $5.85 \pm 0.41$ & $2.09 \pm 0.19$ & $0.90 \pm 0.30$ \\
$\chi^{2} / \mathrm{DF}$ & 1.3 & 1.0 & 1.1 & 0.9 \\
$\mathrm{~N}_{K}$ & $900 \pm 40$ & $396 \pm 28$ & $240 \pm 22$ & $25 \pm 8$ \\
\hline \hline channel & $\nu_{\tau} K \geq 0 \pi^{0} \geq 0 K^{0}$ & $\nu_{\tau} \mathrm{K}$ & $\nu_{\tau} K \pi^{0}$ & $\nu_{\tau} K \pi^{0} \pi^{0}$ \\
\hline $\begin{array}{c}\text { correction } \\
\Delta \mathrm{N}_{K}\end{array}$ & $+67 \pm 24$ & $-60 \pm 22$ & $-20 \pm 8$ & $-14 \pm 4$ \\
efficiency (\%) & 63.8 & 55.6 & 44.0 & 30.3 \\
$\mathrm{~B}_{K}(\%)$ & $1.60 \pm 0.07 \pm 0.12$ & $0.64 \pm 0.05 \pm 0.05$ & $0.53 \pm 0.05 \pm 0.07$ & $0.04 \pm 0.03 \pm 0.02$ \\
\hline
\end{tabular}

Table 2: Results from the $\mathrm{dE} / \mathrm{dx}$ fit and final branching fractions for inclusive and exclusive final states with statistical and systematic uncertainties, respectively. 


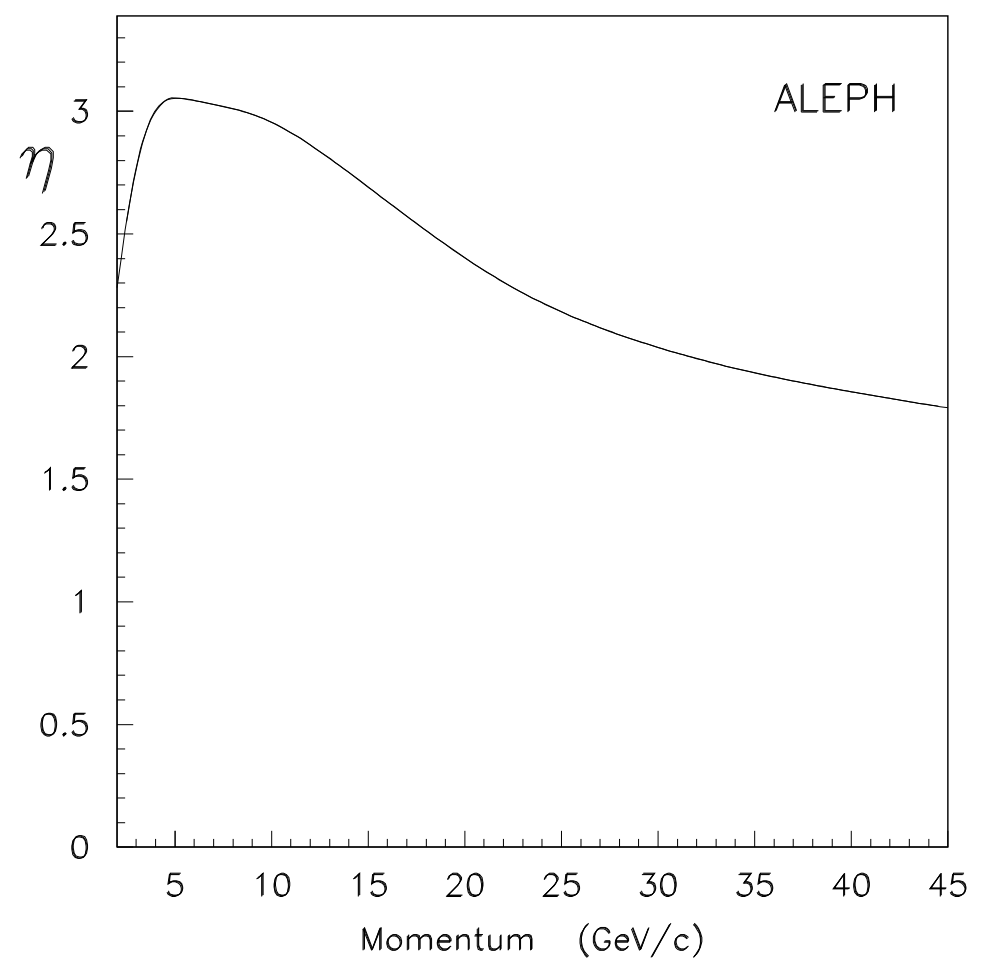

Figure 1: K- $\pi$ separation parameter $\eta$ as a function of momentum. 

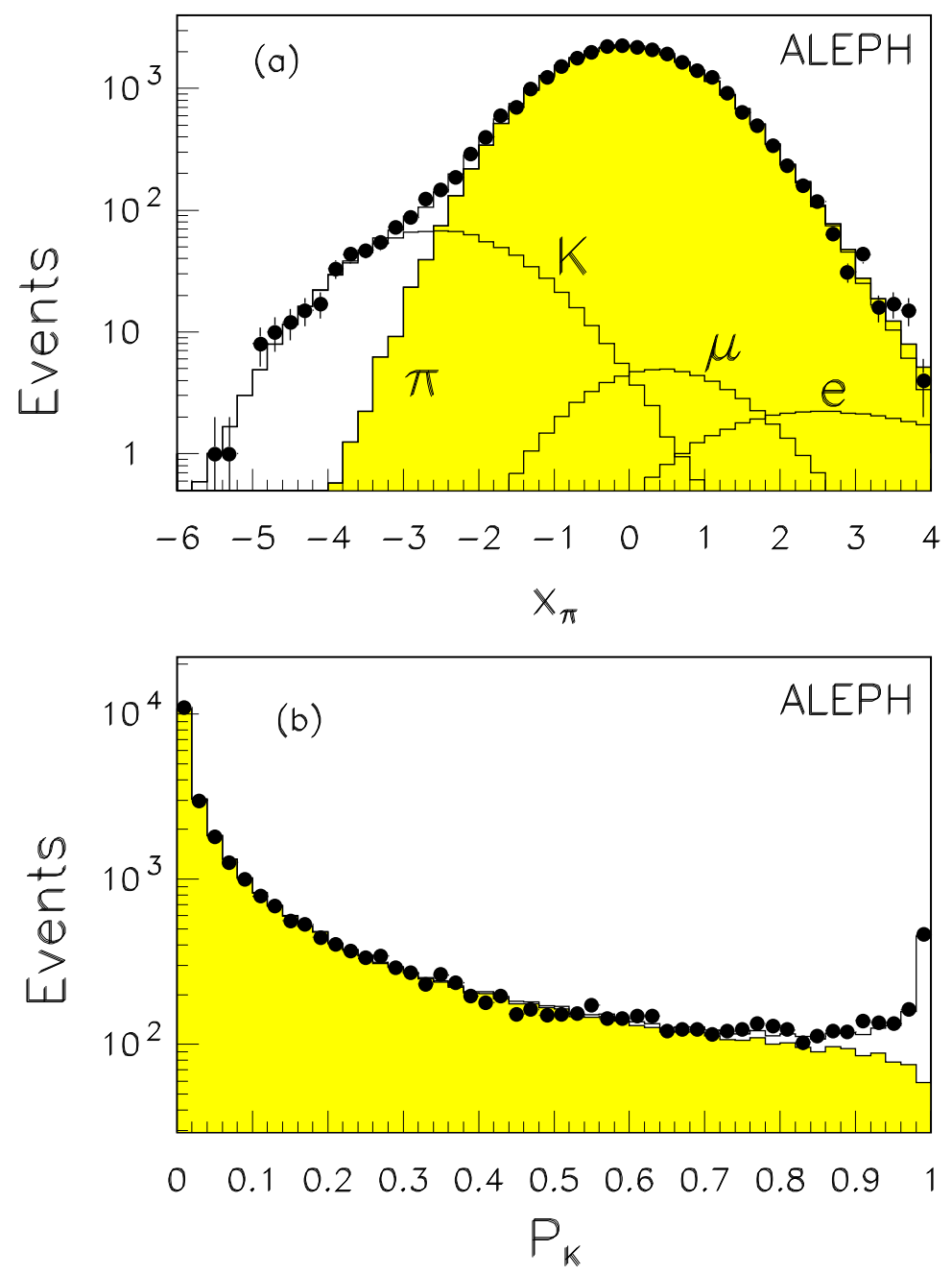

Figure 2: (a) Fit to the $x_{\pi}$ distribution in 1-prong inclusive hadronic $\tau$ decays with the dominant $\pi$ contribution, the $\mu$ and e contaminations and the fitted $\mathrm{K}$ component.(b) Distribution of the estimator $\mathrm{P}_{K}$ for the same sample. The shaded histograms correspond to the sum of all 'background' $\pi, \mu$, e contributions, the K signal being added for the full histogram. 

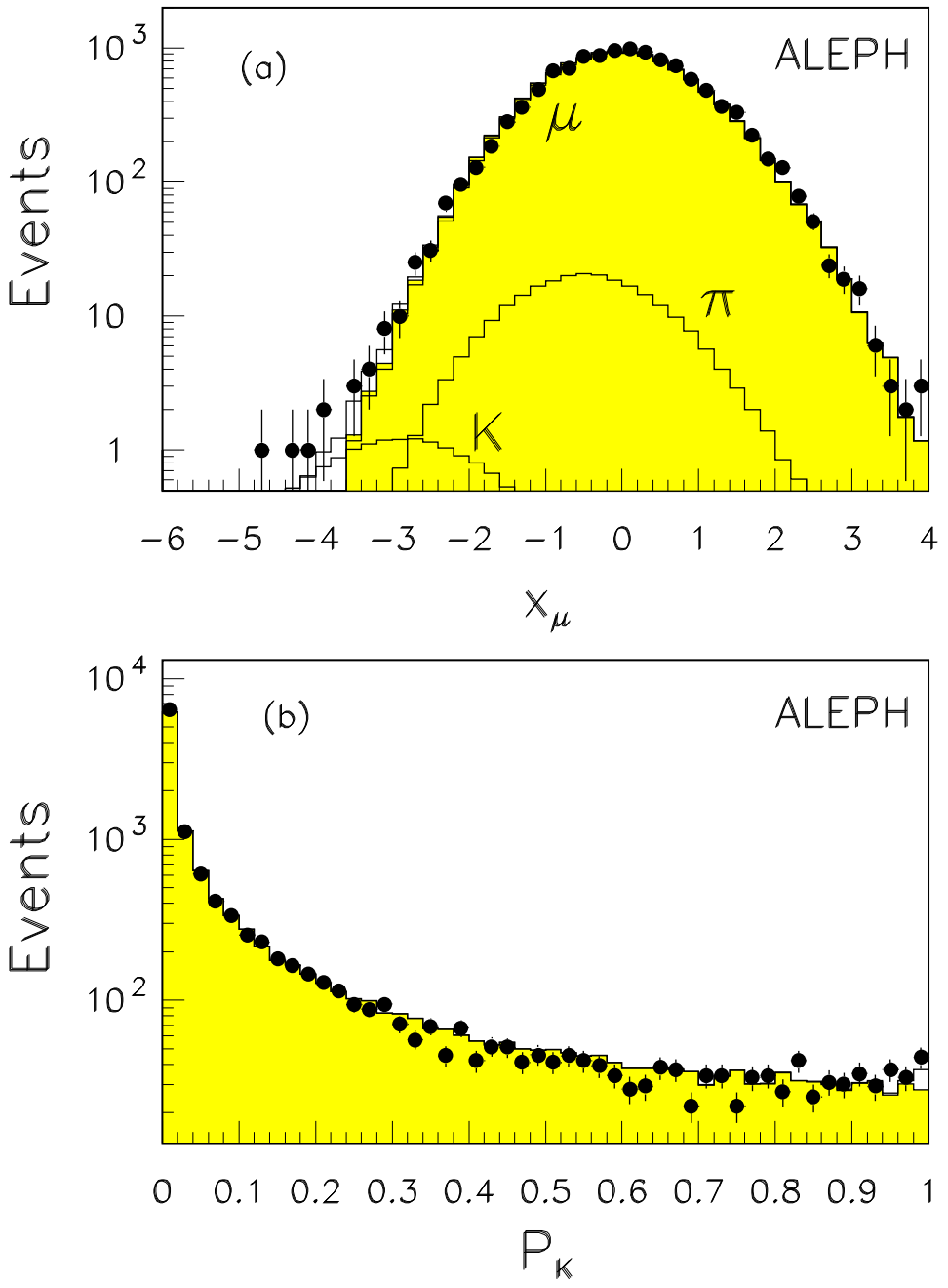

Figure 3: (a) Fit to the $x_{\mu}$ distribution in $\tau \rightarrow \nu_{\tau} \mu \bar{\nu}_{\mu}$ events with $\pi$ and $\mathrm{K}$ contaminations from mis-identification. (b) Distribution of the estimator $\mathrm{P}_{K}$ for the same sample. The shaded histograms correspond to the sum of all 'background' $\pi$, $\mu$ contributions, the $\mathrm{K}$ signal being added for the full histogram. 


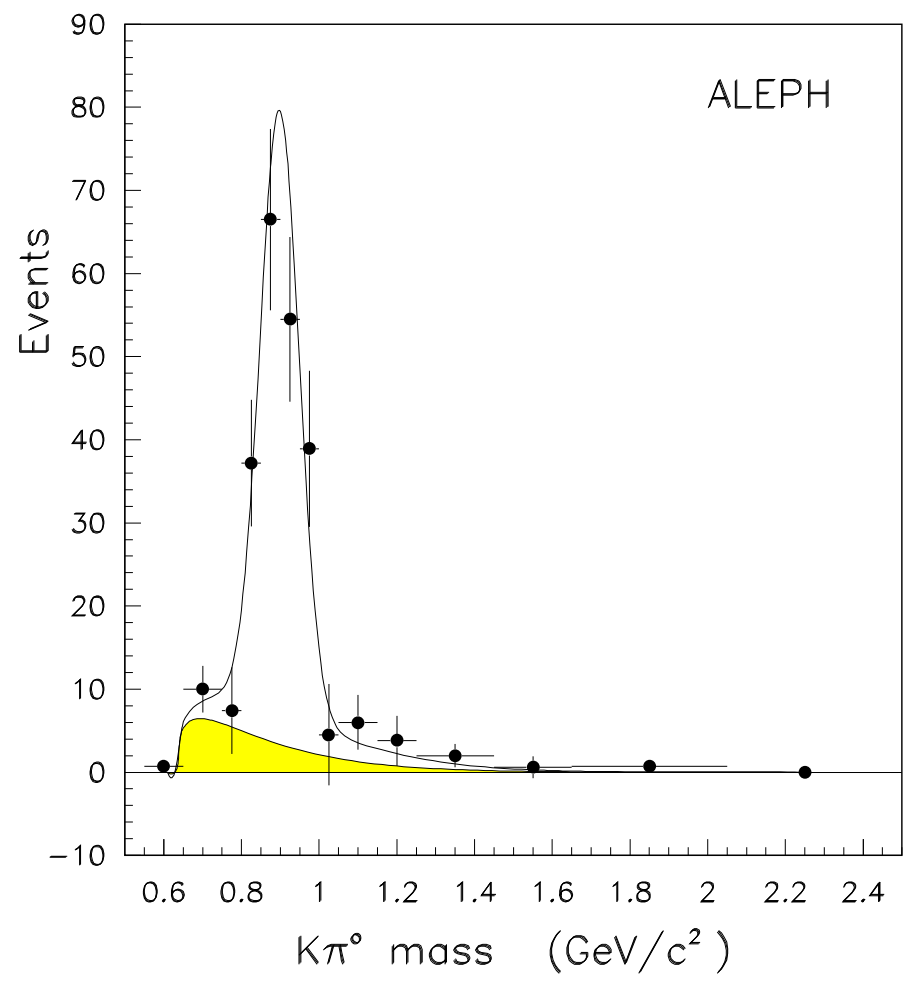

Figure 4: Invariant mass distribution of the $\mathrm{K} \pi^{0}$ final state, as obtained from a $\mathrm{dE} / \mathrm{dx}$ fit in each mass bin ; the Monte Carlo distributions (solid lines) are given for the $\tau \rightarrow \nu_{\tau} K \pi^{0}$ mode and other smaller modes which feed into the selected sample (shaded). 